\title{
Biology, Metastatic Patterns, and Treatment of Patients with Triple-Negative Breast Cancer
}

\author{
Carey K. Anders and Lisa A. Carey \\ Department of Medicine, Division of Hematology-Oncology, University of North Carolina at \\ Chapel Hill
}

\begin{abstract}
Of the estimated 1 million cases of breast cancer diagnosed annually worldwide, it is estimated that over 170,000 will harbor the triple-negative (estrogen receptor/progesterone receptor/HER2negative) phenotype. Most, though not all, triple-negative breast cancers will be basal-like on gene expression micorarrays. The basal-like molecular subtype exhibits a unique molecular profile and set of risk factors, aggressive and early pattern of metastasis, limited treatment options, and poor prognosis. Large population-based studies have identified a higher proportion of triple-negative breast tumors among premenopausal African American women, and a suggestion that increased parity, younger age at first-term pregnancy, shorter duration of breast feeding, and elevated hip-towaist ratio might be particular risk factors. When $B R C A l$ mutation carriers develop breast cancer, it is usually basal-like; given the central role of $B R C A 1$ in DNA repair, this could have profound therapeutic implications. When diagnosed, triple-negative breast cancers illustrate preferential relapse in visceral organs, including the central nervous system. Although initial response to chemotherapy might be more profound, relapse is early and common among triple-negative breast cancers compared with luminal breast cancers. The armamentarium of "targeted therapeutics" for triple-negative breast cancer is evolving and includes strategies to inhibit angiogenesis, epidermal growth factor receptor, and other kinases. Finally, the positive association between triple-negative breast cancer and BRCA mutations makes inhibition of poly(adenosine diphosphate-ribose) polymerase -1 an attractive therapeutic strategy that is in active study.
\end{abstract}

\section{Keywords}

Basal-like breast cancer; BRCA1; BSI-201; Cetuximab; Cytokeratin 5/6; PARP1

\section{Introduction}

Over the past decade, the landscape of breast cancer has changed. We have discovered that breast cancer, once thought to be a rather homogenous disease, is not a single disease process but rather a compilation of several different and unique subtypes as defined via gene expression analysis. ${ }^{1}$ Microarray techniques have divided breast cancer into several intrinsic subtypes: luminal A, luminal B, HER2-enriched, and the "basal-like" subtype (Figures 1A

\footnotetext{
Address for correspondence: Lisa A. Carey, MD, Department of Medicine, Division of Hematology/Oncology, University of North Carolina Lineberger Comprehensive Cancer Center, Physician's Office Building, 3rd Floor, 170 Manning Dr, Campus Box 7305 , Chapel Hill, NC 27599, Fax: 919-966-6735; lisa_carey@med.unc.edu.

Dr. Anders and Dr. Carey have no relevant relationships to disclose.

This article includes the discussion of investigational and/or unlabeled use of drugs, including the use of BSI-201, AG014699, AZD2881, cetuximab, irinotecan, carboplatin, cisplatin, dasatinib, and vorinostat for the treatment of patients with breast cancer, and carboplatin in combination with gemcitabine or albuminbound paclitaxel and bevacizumab in combination with docetaxel or capecitabine for the treatment of patients with metastatic breast cancer.
} 
and $1 \mathrm{~B})$. Both luminal A and B are clinically characterized by expression of hormone receptor-related genes, whereas both HER2-enriched and the "basal-like" subtypes are less likely to express either estrogen receptor (ER) or progesterone receptor $(\mathrm{PgR})$. Moreover, the "basal-like" subtype, one of the most clinically aggressive of the subtypes, is more commonly negative for all 3 markers_ER, PgR, and HER2_-hence the "triple-negative" phenotypic classification. ${ }^{2,3}$

Of the estimated 1 million cases of breast cancer diagnosed worldwide in 2008, it is estimated that 172,695 will harbor the triple-negative phenotype. ${ }^{4}$ Triple-negative breast cancer is currently receiving a tremendous and appropriate amount of research attention given its unique biology, overall poor prognosis, aggressive and early pattern of metastases, and relative lack of therapeutic targets when compared with endocrine-sensitive and HER2positive breast cancers. This review will focus on molecular features, risk/epidemiologic factors, patterns of metastatic spread, prognostic implications, novel "targets," and emerging therapeutic strategies for this clinically challenging and aggressive entity.

\section{Pathologic and Molecular Features of Triple-Negative Breast Cancer}

Triple-negative breast cancer has both unique pathologic and molecular characteristics (Table 1).2,5,6 Although frequently referred to interchangeably, it is important to clarify that the terms "triple negative" and "basal-like" are not completely synonymous, illustrating an approximately $20 \%-30 \%$ discordance across several studies. ${ }^{5,7-9}$ The term triple negative refers to the immunohistochemical classification of breast tumors lacking ER, PgR, and HER2 protein expression, whereas the basal-like subtype is defined via gene expression microarray analysis. ${ }^{2,3}$ To date, the basal-like classification is available only in the research setting; thus, the triple-negative phenotype currently serves as a reliable surrogate in the clinical arena.

Several investigators have sought to identify clinically useful markers characteristic of the basal-like breast cancer subtype. Nielsen et al collected a series $(n=21)$ of known basal-like breast tumors as determined via cDNA microarray and examined the significance of protein expression patterns via tissue microarray. ${ }^{5}$ Results indicated that the majority of basal-like breast tumors illustrated low-to-absent expression of ER and HER2, with high expression of HER1 (epidermal growth factor receptor [EGFR]), basal cytokeratin 5/6, and c-Kit. Interestingly, a survival analysis of over 900 cases illustrated a shorter disease-specific survival among cases that expressed the basal cytokeratins 5/6 and 17. In addition, expression of HER1 was a significant independent negative prognostic factor (relative risk [RR], 1.54; $P=.017)$ when applying the available clinical variables of tumor size $(\mathrm{RR}, 1.12)$ and nodal status (RR, 2.10). Finally, expression of c-Kit via tissue microarray was not a predictor of patient outcome.

A second investigation of the histologic and immunophenotypic characterizations of basallike breast tumors corroborated the above findings. Livasy et al evaluated 56 tumors with known microarray profiles, 23 of which were basal-like. ${ }^{6}$ Results indicated that the basallike tumors were grade 3 , ductal $(21 / 23)$ or metaplastic $(2 / 23)$ carcinomas and frequently exhibited geographic necrosis (17/23), a pushing border of invasion (14/23), and a stromal lymphocytic response (13/23). All basal-like tumors tested were ER and HER2 negative and illustrated immunoreactivity for vimentin (17/18), luminal cytokeratin 8/18 (15/18), EGFR (13/18), and cytokeratin 5/6 (11/18). Interestingly, myoepithelial markers (ie, smooth muscle actin, p63, and CD10) were infrequently positive. Consistent with previous reports, the most consistent immunophenotype seen in the basal-like tumors was negativity for ER and HER2 and positivity for vimentin, EGFR, cytokeratin 8/18, and cytokeratin 5/6. 
In addition to a characteristic immunophenotypic profile, triple-negative breast cancer has been associated with several aggressive pathologic features. As part of the Carolina Breast Cancer Study, breast cancer subtype, as defined via immunophenotypic classifications, and associations with tumor size, axillary lymph node status, mitotic index, nuclear pleomorphism, combined grade, and p53 mutation status were all examined. ${ }^{10}$ Compared with the endocrine-sensitive, luminal A (ER and/or PgR positive/HER2 negative) breast tumors, basal-like (ER/PgR/HER2-negative) breast tumors harbored a higher number of TP53 mutations (44\% vs. 15\%; $P<.001$ ), higher mitotic index (odds ratio [OR], 11.0; 95\% CI, 5.6-21.7), more marked nuclear pleomorphism (OR, 9.7; 95\% CI, 5.3-18.0), and higher combined grade (OR, 8.3; 95\% CI, 4.4-15.6). Matos et al also illustrated that among 168 invasive breast cancers, $7.6 \%$ of which were basal (defined as ER/HER2 negative), the majority of basal-like tumors were grade III and illustrated a high proliferation rate. ${ }^{11}$ In addition, these tumors were more commonly p-cadherin/p63 negative and cytokeratin 5 positive.

Finally, an association between the triple-negative phenotype and breast cancers harboring germline mutations in the BRCAl gene has been well-described. The BRCAl gene, located on chromosome 17 (17q21) and often termed the "caretaker of the genome," is responsible for both inherent DNA damage-sensing processes and DNA repair mechanisms. Mutations in this important gene confer an approximately $80 \%$ lifetime risk of breast cancer among carriers.12,13 The large majority of BRCA1-associated breast cancers express the triplenegative phenotype in addition to "basal-like" cytokeratins (CK 5, 14, 17) and HER1/EGFR. $14^{-17}$ In addition, gene expression studies further support this connection, because BRCA1mutated breast tumors typically cluster within the basal-like subtype. ${ }^{3}$ The observed association between BRCA1 mutation status and triple-negative breast cancer provides a novel therapeutic approach incorporating agents (ie, poly[adenosine diphosphate-ribose] polymerase [PARP]1 inhibitors) capable of further inhibiting DNA repair mechanisms, which will be discussed in more detail herein.

\section{Risk Factors and Epidemiologic Features}

In addition to a distinct molecular and pathologic profile, the epidemiology and risk factors associated with triple-negative breast cancer are distinct, especially when compared with endocrine-sensitive luminal breast tumors. The Carolina Breast Cancer Study, a populationbased, case-control study aimed at determining clinical associations and distributions across distinct breast cancer subtypes, has refined our understanding of the epidemiologic and risk factors associated with triple-negative breast cancer. ${ }^{10}$ In the initial study of women diagnosed with invasive breast cancer, the prevalence of breast cancer subtypes within racial and menopausal subsets were determined. Immunohistochemical staining was used to classify specific subtypes in approximately 500 tumors, and "basal-like" tumors were defined as triple negative (ER/PgR/HER2 negative) and cytokeratin 5/6 positive and/or HER1 positive. Results indicated that those with basal-like tumors were more likely to be African American compared with non-African American (26\% vs. 16\%) and premenopausal compared with postmenopausal ( $24 \%$ vs. $15 \%)$. There was a particularly high prevalence of basal-like tumors among premenopausal African American women compared with postmenopausal African American women and non-African American women of any age (39\% vs. $14 \%$ and $16 \% ; P<.001$ ). The observation that triple-negative breast cancers more commonly arise in younger African American women has been confirmed in several additional studies, although the exact cause for this association is not yet fully understood. 18,19

An expansion of the Carolina Breast Cancer Study sought to examine commonly reported breast cancer risk factors among 1424 cases of invasive and in situ breast cancer compared 
with > 2000 controls (Table 2). ${ }^{20}$ As expected, for patients with luminal A breast cancer (defined as ER positive and/or PgR positive and HER2 negative via immunohistochemistry), risk was inversely associated with increased parity and younger age at first-term pregnancy. In contrast, for basal-like breast cancer, risk was increased with parity and younger age at first term full-term pregnancy. In addition, a reduced risk for basal-like breast cancer was observed among those with a longer duration of breast-feeding, increasing number of children breast-fed, and increasing number of months breast-feeding. This observation was not seen among those diagnosed with luminal A breast cancer. Among postmenopausal women with an elevated waist-to-hip ratio, an increased risk for luminal A breast cancer was observed. This observation held true for both pre- and post-menopausal women with regard to basal-like breast cancer risk. Strikingly, the authors concluded that if these associations hold true among younger African American women who exhibit the highest percentage of basal-like breast cancer risk factors, close to two thirds of basal-like breast cancers could be prevented by promoting breast-feeding and reducing abdominal adiposity. Similarly, the Polish Breast Cancer Study reported differential risk factor indices by breast cancer subtype. ${ }^{21}$ In this population-based study, increasing age at menarche was associated with reduced risk of basal-like, but not luminal, cancers, whereas increasing body mass index among premenopausal women was associated with a reduced risk of luminal, but not basal-like, cancers. Taken together, these studies illustrate that risk factors vary by subtype and must be taken into account as prevention strategies are planned and investigated.

\section{Prognosis and Patterns of Metastatic Spread}

The earliest gene expression profiling studies identified the basal-like subtype as a poor prognosis subgroup with regard to relapse-free survival $(P<.01)$ and overall survival (OS; $P$ $<.01$ ); Figures $2 \mathrm{~A}$ and Figure $2 \mathrm{~B}$ ). ${ }^{2}$ Results were confirmed across additional and independent data sets illustrating a clear discrimination between tumors that illustrated high expression of genes classified as luminal A (endocrine-sensitive tumors) compared with those lacking expression of these genes (ie, basal and HER2-positive subtypes). Prognostic significance was again verified. Kaplan-Meier analysis demonstrated that patients with tumors classified as basal-like or HER2 positive showed much shorter disease-free survival (DFS) intervals compared with patients with luminal A tumors. ${ }^{3}$

To further define prognosis of patients classified as "triple negative" via clinical parameters (ER/PgR/HER2 negative), a large cohort study of > 1600 women diagnosed with and treated for invasive breast cancer in Toronto from 1987 to 1997 was performed. ${ }^{22}$ Among the $11.2 \%$ of patients diagnosed with triple-negative breast tumors, both the likelihood of distant recurrence (hazard ratio $[\mathrm{HR}], 2.6 ; P<.0001$ ) and death from breast cancer within 5 years of diagnosis (HR, 3.2; $P<.0001$ ) were higher compared with non-triple-negative phenotypes. Patterns in both distant recurrence and breast cancer-specific mortality were seen during the first 5-7 years following diagnosis (peaking at 3 years and quickly declining), but not thereafter. Interestingly, this study also reported that triple-negative breast tumors were more likely to be detected through clinical examination than through imaging (ie, mammography, ultrasound; $36 \%$ vs. $19.6 \% ; P=.0008$, respectively) when compared with breast cancers of other phenotypes. This observation, in addition to a second study reporting that triplenegative breast tumors were more likely to present as "interval cancers" between regular mammograms, ${ }^{23}$ might reflect either a more aggressive and rapid growth rate or intrinsic differences in the density of breast tissue among women diagnosed with triple-negative breast cancer.

A study evaluating response to neoadjuvant chemotherapy among $>1000$ patients treated at The University of Texas M. D. Anderson Cancer Center from 1985 to 2004 corroborated the above prognostic findings. ${ }^{24}$ Results demonstrated decreased 3-year progression-free 
survival (PFS; $P<.0001)$ and 3-year OS $(P<.0001)$ rates for triple-negative compared with non-triple-negative breast cancer. Interestingly and consistent with previous reports, recurrence and death rates were higher for triple-negative breast cancer only in the first 3 years following diagnosis. The observed patterns speak to the early and aggressive nature of recurrences among patients with triple-negative breast cancer.

In addition to patterns observed in the timing of recurrence, preferential site of relapse has also been identified among triple-negative/basal-like breast tumors. Dent et al reported that few women with triple-negative breast cancer experience a local recurrence before a distant recurrence. ${ }^{22}$ More specifically, Liedtke et al reported that patients with triple-negative breast cancer have higher rates of recurrence in visceral organs and soft tissue, with lower rates of bone disease, compared with hormone-sensitive counterparts $(P=.027) .{ }^{24}$ Similar results were reported among 344 lymph node-negative primary breast tumors subject to molecular classification. ${ }^{25}$ Bone relapse was most abundantly seen among patients with breast tumors classified as the luminal subtype but were found less than expected in the basal-like subtype. For lung and brain relapse, the opposite was true; both were observed more commonly among patients with basal-like breast tumors.

An increasing interest in brain metastases among patients with triple-negative breast cancer has emerged. Recent studies indicate an increased incidence and uniquely aggressive nature of brain metastases arising from triple-negative breast cancer. $26^{-} 28$ A retrospective analysis of > 3000 patients treated in Europe between 1989 and 2006 illustrated that 338 (10.6\%) had triple-negative breast cancer. ${ }^{26}$ Of the 80 patients who developed brain metastases over a 51-month median follow-up period, 19 (23.75\%) were diagnosed with the triple-negative phenotype. Multivariate analysis indicated that the triple-negative phenotype conferred the highest risk for developing brain metastases compared with other types of breast cancer (odds ratio [OR], 4.16; $P<.001$ ). The median interval between primary diagnosis and occurrence of brain metastases (22 months vs. 51 months; OR, 2.7; $P<.0001)$ and OS after occurrence of brain metastases ( 4 months vs. 8 months; $P=$ not significant) was shorter among those with triple-negative breast cancer compared with other types.

A recent study of 116 patients treated for triple-negative metastatic breast cancer (MBC) at Dana-Farber Cancer Institute between January 2000 and June 2006 characterized outcomes of patients with triple-negative MBCs, including the risk and clinical consequences of central nervous system (CNS) recurrence. ${ }^{27}$ Recurrence characteristics indicated a high incidence of visceral metastases, both lung and liver metastasis, at initial metastatic diagnosis (41\% and 29\%) and initial/subsequent recurrence (64\% and 50\%). Strikingly, $14 \%$ of the patients were diagnosed with CNS metastases at initial metastatic diagnosis, and $46 \%$ were diagnosed with CNS involvement during their metastatic course. Median survival following diagnosis of CNS metastases was 4.9 months. Age- and race-adjusted death rate for the patients with a CNS metastasis at first presentation was 3.4 times (95\% CI, 1.9 times-6.1 times) that of those with no CNS lesion at first metastatic presentation. At the time of CNS diagnosis, extracranial systemic disease was stable or responding to systemic therapy in a minority of patients $(n=9 ; 17 \%)$. On the contrary, $83 \%$ of patients were concurrently diagnosed with CNS metastases and new or progressive systemic metastases.

Interestingly, the natural history of brain metastases arising from triple-negative breast cancer and HER2-enriched breast cancer, a subtype also prone to CNS disease, differs with regard to both disease control and likelihood of isolated CNS progression. A retrospective analysis of 122 women treated with the HER2-targeted monoclonal antibody (MoAb) trastuzumab at Dana-Farber Partners Cancer Care from 1998 to 2000 indicated that $34 \%$ of patients (95\% CI, 26\%-44\%) were diagnosed with CNS metastases at a median of 16 months following diagnosis of MBC and 6 months from the beginning of trastuzumab 
therapy. ${ }^{29}$ In contrast to the triple-negative phenotype, $50 \%$ of patients were responding or had stable disease (SD) while receiving trastuzumab at the time of diagnosis of CNS metastases. The median survival period after CNS metastases was 13 months. Among the 41 patients diagnosed with CNS metastases, approximately $50 \%$ of patients died of progressive CNS disease. The authors concluded that the isolated CNS progression observed among the HER2-positive, trastuzumab-treated population is either because of predilection for the CNS by HER2-positive tumor cells and/or poor penetration of the CNS by trastuzumab or to improved visceral disease control, leading to a longer life and onset of late tumor spread to the CNS, although this is not completely understood. This apparent contrast between the natural history of these 2 aggressive breast cancer subtypes serves to highlight the systemic nature of triple-negative breast cancer and further supports the need for effective and novel therapeutic agents capable of crossing the blood-brain barrier, an area of fertile and active research, in an effort to control both aggressive intracranial and extracranial advanced triplenegative breast cancer.

\section{Response to Standard Chemotherapeutic Approaches}

A paradoxical finding is that triple-negative breast tumors often have a more profound initial response to chemotherapy compared with other phenotypes (ie, ER and/or HER2 positive) despite poorer overall survival. This observation is supported by several studies in the neoadjuvant setting.24,30,31 A prospective study conducted at The University of Texas M. D. Anderson Cancer Center sought to characterize the relationship between molecular class (ie, basal-like, luminal, HER2 defined by clustering using the intrinsic gene set) and chemotherapy sensitivity among 82 patients with early-stage breast cancer treated with neoadjuvant anthracycline- and taxane-based chemotherapy.31 Results indicate pathologic complete response (pCR) rates were reported to differ significantly among the molecular classes of breast cancer. The basal-like and HER2-positive subgroups were associated with the highest rates of pCR (45\% [95\% CI, 24\%-68\%] and 45\% [95\% CI, 23\%-68\%], respectively). Conversely, pCR among luminal tumors was substantially lower (6\%; $95 \%$ CI, $1 \%-21 \%$ ). Interestingly, differential gene expression analysis between the basal-like and HER2-positive tumors revealed no overlap in genes associated with pCR, suggesting that the molecular mechanisms of chemotherapy sensitivity might vary between these 2 ER-negative subtypes.

Two studies examined not only initial response of triple-negative breast tumors to neoadjuvant chemotherapy but also the relationship of response to chemotherapy with overall outcome. A prospectively maintained dataset of 107 patients with breast cancer treated with neoadjuvant anthracycline-based (doxorubicin/cyclophosphamide [AC]) chemotherapy was examined at the University of North Carolina in order to address these questions. ${ }^{30}$ Utilizing immunohistochemical profiles to define breast cancer subtypes (ie, basal [hormone receptor/HER2 negative], HER2 [hormone receptor negative/HER2 positive], luminal B [hormone receptor/HER2 positive], luminal A [hormone receptor positive/HER2 negative]), results indicated that clinical response to anthracycline-based chemotherapy was higher among the HER2-positive (70\%) and basal-like (85\%) subtypes compared with the luminal subtypes $(47 \% ; P<.0001)$. Although pCR rates were higher among the HER2-positive/ER-negative (36\%) and basal-like (27\%) tumors compared with luminal subtypes (7\%; $P=.01$ ), patients with HER2-positive/ER-negative and basal-like tumors experienced inferior distant DFS and OS ( $P=.04$ and $P=.02$, respectively).

Liedtke et al subsequently conducted an analysis of a prospectively collected clinical database, including 1118 patients who received neoadjuvant chemotherapy at The University of Texas M. D. Anderson Cancer Center from 1985 to 2004 for stage I-III breast cancer. ${ }^{24}$ All patients, of which 255 (23\%) had triple-negative breast cancer, had received at 
least 1 cycle of chemotherapy. Overall, 163 patients (15\%) achieved a pCR. Multivariate analysis illustrated increased pCR rates among triple-negative compared with non-triplenegative breast cancer patients (OR, $1.53 ; P=.034)$. Again, despite higher pCR rates, triplenegative status compared with non-triple-negative status conferred a poorer 3-year PFS rate (63\% vs. $76 \%$, respectively; HR, $1.86 ; P<.0001)$. The higher rates of relapse among patients with triple-negative breast cancer appears attributable to residual disease at the time of surgery, highlighting a need for either more effective neoadjuvant therapies or defined adjuvant, residual disease protocols.

The inherent chemotherapy sensitivity of triple-negative breast cancer is not restricted to the neoadjuvant setting. In the adjuvant setting, a retrospective review of a subset of patients enrolled in the Cancer and Leukemia Group B (CALGB) 9344 clinical trial $(n=1322)$ indicated that the addition of a taxane (paclitaxel) to anthracycline-based chemotherapy provided the greatest benefit to patients with either HER2-positive or ER/HER2-negative breast tumors. ${ }^{32}$ Although not designed to test this definitively, the addition of paclitaxel did not appear to substantially benefit patients with HER2-negative/ER-positive cancers. Additionally, the landmark Intergroup C9741/CALGB trial 9741 established both a DFS ( $P$ $=.012)$ and an OS benefit $(P=.049)$ for dose-dense (every 2 weeks) anthracycline/taxanebased chemotherapy compared with conventional scheduling (every 3 weeks) in the setting of node-positive, early-stage breast cancer. ${ }^{33}$ Interestingly, an exploratory analysis of both DFS and OS by ER status and dose density suggested a larger absolute benefit in ERnegative disease $(P=.014$ and $P=.039$, respectively; $P=$ not significant in ER-positive disease). Although this evaluation represents a retrospective subset analysis, these results are thought-provoking and continue to support the inherent chemotherapy sensitivity observed among endocrine-insensitive breast tumors.

As described above, triple-negative breast cancer is highly responsive to primary anthracycline and anthracycline/taxane chemotherapy; however, a high risk of relapse remains if the tumor is not eradicated. Both preclinical and clinical studies indicate that tumors with BRCA1 dysfunction, the majority of which are triple negative, harbor deficient double-stranded DNA break repair mechanisms and are sensitive to DNA-damaging chemotherapeutic agents, such as platinum agents (ie, cisplatin and carboplatin). ${ }^{34,35}$ The association between BRCA1 dysfunction and triple-negative breast cancer has led to several neoadjuvant/adjuvant and metastatic studies evaluating platinum agents in the setting of triple-negative breast cancer.36 39 Although efficacy has yet to be compared with standard anthracycline/taxane-based therapies, platinum agents are quickly emerging as the chemotherapy "backbone" of choice when combined with novel agents.

\section{Novel "Targeted" Therapeutic Agents}

Given the absence of known "targeted therapy" for triple-negative breast cancer, investigators have been fervently investigating molecular targets among triple-negative breast tumors in order to advance the development of novel therapeutic agents aimed at treating this clinically aggressive phenotype. Molecular entities characteristic of triplenegative breast cancer have included expression of HER1 and c-Kit; mutation/perturbation of p53; activation of BRCA1-associated pathways, namely PARP1; and activation of protein kinase components of the mitogen-activated protein kinase and protein kinase B (Akt) pathways. ${ }^{3,5}, 8,38,40,41$ In addition, GRB7, a calmodulin-binding protein that binds phosphorylated tyrosine residues (ie, EGFR, HER2) and the small heat shock protein $\alpha$ basic-crystallin ( $\alpha \mathrm{B}$-crystallin) have been associated with inferior outcome among patients diagnosed with triple-negative and basal-like breast tumors.42,43 Finally, preclinical models have illustrated re-expression of ER- $\alpha$ in ER-negative breast cancer cell lines following treatment with histone deacetylase (HDAC) inhibitors (ie, trichostatin A and Scriptaid), 
thereby potentially opening up an avenue for therapy with endocrine agents.44 Strategies targeting the EGFR and Src pathways, PARP1 and HDAC inhibitors, and antiangiogenic agents are currently in clinical trials and are described herein, providing glimpses into the role, if any, of these and other strategies (Table 3). ${ }^{30-32,36-39,42,44-51}$

\section{Antiangiogenesis Strategies: Bevacizumab}

Both laboratory and clinical evidence supports a central role for angiogenesis in the progression of breast cancer, and strategies that inhibit tumor angiogenesis have shown promise in the setting of advanced disease. The landmark randomized phase III E2100 study evaluating the addition of bevacizumab, a MoAb targeting vascular endothelial growth factor-A, to paclitaxel reported an improvement in overall response rate and PFS compared with paclitaxel alone (36.9\% vs. $21.2 \% ; P<.001$ and 11.8 months vs. 5.9 months; HR, 0.60; $P<.001$, respectively). ${ }^{45}$ Overall survival rate was similar between both groups (26.7 months vs. 25.2 months; HR, $0.88 ; P=.16$ ). Interestingly, multivariate analysis indicated that the benefit of incorporating bevacizumab was also seen in patients with ER- and PgRnegative disease, the large majority (> 90\%) of which were also HER2 negative ( 8.8 months vs. 4.6 months; HR, 0.53 ; 95\% CI, 0.40-0.70), suggesting a targeted drug with efficacy in this subtype. Although the final decision generated controversy, the US Food and Drug Administration granted accelerated approval on February 22, 2008, for incorporation of bevacizumab with paclitaxel for the first-line treatment of HER2-negative MBC.

Two additional studies have confirmed the activity of bevacizumab in the setting of advanced breast cancer. The randomized, doubleblind, placebo-controlled, phase III AVADO (Avastin ${ }^{\circledR}$ and Docetaxel in Metastatic Breast Cancer) study presented at the 2008 annual American Society of Clinical Oncology (ASCO) meeting investigated the combination of bevacizumab and docetaxel as first-line therapy for patients with locally recurrent breast cancer or MBC. ${ }^{46}$ Results indicated an improvement in both overall response rate and PFS among patients treated with combination therapy with bevacizumab $15 \mathrm{mg} / \mathrm{kg}$ compared with those treated with docetaxel and placebo $(44.4 \%$ vs. $63.1 \% ; P=$. 0001 and 8.0 months vs. 8.8 months; HR, $0.61 ; 95 \%$ CI, 0.48-0.78, respectively). As observed in E2100, subgroup analysis indicated that the ER- and PgR-negative subgroup continued to derive a significant benefit (HR, 0.60). Overall survival differences were not observed; however, the data are immature (median, 10.2 months; range, 0-17.5 months). Finally, Genentech issued a press release in November 2008 announcing that the placebocontrolled phase III RIBBON-1 study met its primary endpoint of improving PFS in women with MBC. RIBBON-1 randomized 1237 patients with chemotherapy-naive disease to receive a taxane, anthracycline, or capecitabine with or without bevacizumab. Efficacy and safety results were reported at the 2009 ASCO annual meeting. The E2100, AVADO, and RIBBON-1 studies all indicate that antiangiogenic agents appear to show benefit in selected patients with MBC within and across subtypes; however, identification of the subsets expected to derive the greatest benefit has yet to be fully elucidated and remains an active area of research interest.

\section{PARP1 Inhibition: BSI-201 and Others}

Poly(adenosine diphosphate-ribose) polymerases are involved in the molecular events leading to cell recovery from DNA damage. When PARP1, the most abundant member of the PARP family, is inhibited, double-strand DNA breaks accumulate and, under normal conditions, are repaired via homologous recombination. ${ }^{52}$ As described above, one of the central roles of $B R C A 1$ is to repair DNA double-strand breaks via homologous recombination; thus, investigators have hypothesized that the inhibition of PARP, in combination with DNA-damaging chemotherapeutics, would render tumors lacking BRCAI 
function exquisitely sensitive-a hypothesis that has borne out in both the preclinical and clinical arenas. $47,48,53,54$

BRCA1-mutated, sporadic triple-negative breast tumors, and basal-like breast cancers share several molecular, phenotypic, and prognostic features. ${ }^{35,52,55}$ The tight association between $B R C A 1$ mutations and the triple-negative/basal-like subtype has raised the question as to whether $B R C A 1$ loss of function, through other mechanisms, participates in triple-negative phenotype in sporadic tumors and if a shared therapeutic approach might be appropriate. An ongoing randomized phase II study is evaluating the clinical benefit of adding PARP1 inhibition, BSI-201 (BIPAR Sciences, Inc.), to DNA-damaging doublet chemotherapy (gemcitabine and carboplatin) in the setting of triple-negative advanced breast cancer. ${ }^{39}$ An interim analysis presented at the 2008 San Antonio Breast Cancer Symposium reported safety of combination therapy and, interestingly, gene expression profiling (multiplex quantitative reverse-transcriptase polymerase chain reaction) results from the first 28 patients enrolled confirmed that the breast tumors illustrated significant upregulation of PARP1 compared with normal breast tissue. A recent update at the 2009 ASCO annual meeting reported that the addition of BSI-201 significantly increased overall response rate (48\% vs. $16 \% ; P=.002)$, clinical benefit rate $(62 \%$ vs. $21 \% ; P=.0002)$, median PFS $(6.9$ months vs. 3.3 months; $P<.0001)$, and median OS (9.2 months vs. 5.7 months; $P=.0005)$ in patients with metastatic triple-negative breast cancer. ${ }^{56}$ A Cancer Research United Kingdom-sponsored study is currently evaluating the PARP inhibitor AG014699 (Pfizer, Inc.) in the setting of BRCA1/2-mutated locally advanced or metastatic breast or ovarian cancer. AZD2881 (Kudos Pharmaceuticals, a subsidiary of AstraZeneca) is being studied as a single agent (phase II) in the setting of BRCA-mutated advanced ovarian cancer and in either $B R C A$-mutated or triple-negative breast cancer. AZD2881 is also being studied in combination with carboplatin (phase I) in the setting of BRCA-mutated or hereditary metastatic breast or ovarian cancer. Efficacy results from several of these trials are anticipated in 2009.

\section{Inhibition of Epidermal Growth Factor Receptor: Cetuximab}

EGFR/HER1 is perhaps the most well-known protein overexpressed among triple-negative breast cancer for which several MoAbs and small-molecular inhibitors exist. A multicenter randomized phase II study of the anti-EGFR MoAb cetuximab alone and in combination with carboplatin was performed to determine benefit in the setting of triple-negative MBC. ${ }^{57}$ Patients in arm 1 received single-agent cetuximab $250 \mathrm{mg} / \mathrm{m}^{2} /$ week $\left(400-\mathrm{mg} / \mathrm{m}^{2}\right.$ loading dose) with carboplatin area under the curve of 2 weekly for 3 of 4 weeks added upon progression; those in arm 2 received cetuximab and carboplatin (same dosing) throughout study enrollment. Updated and final results in this pretreated population indicate a clinical benefit rate $(\mathrm{CR}+$ partial response $[\mathrm{PR}]+\mathrm{SD})$ of $10 \%$ for single-agent cetuximab $(\mathrm{n}=31)$ and $31 \%$ for patients treated with combination therapy. Recurrence occurred rapidly among those with progressive disease; $24 \%$ occurred within 4 weeks, and $48 \%$ occurred within 8 weeks. Overall time to progression was 2 months, and median overall survival was 12 months, illustrating the aggressive nature of this disease. Interestingly, 2 patients treated with cetuximab alone (arm 1) sustained a prolonged PR for $>40$ weeks. Pharmacodynamic studies on paired biopsies from 16 patients (pre- and 1-2 weeks post-therapy) examining gene expression activity of the EGFR pathway indicated that 12 of 16 had active EGFR pathway expression. Despite this activity, anti-EGFR therapy downregulated EGFR pathway expression in only 4 , and all clinical activity was in this cohort. ${ }^{36}$ The mechanism of ongoing pathway activation in the setting of therapeutic inhibitors raises interesting questions about the nature of resistance and is an area of great activity across all targeted therapies. Embedded correlative studies such as this are imperative to foster the identification of alternate mechanisms of activation and resistance in patients with triple- 
negative and other subtypes of breast cancer who might derive the greatest benefit from targeted therapies.

In a related study, O'Shaughnessy et al reported a randomized phase II study of weekly irinotecan/carboplatin (IC) with or without cetuximab (ICE) in 150 patients with unselected MBC. ${ }^{38}$ Objective response was $28 \%$ with IC versus $33 \%$ with ICE; however, the triplenegative subset appeared to benefit more, with responses of $30 \%$ versus $49 \%$, respectively. There was increased toxicity with the addition of cetuximab; most notably, grade $3 / 4$ diarrhea and fatigue occurred in $35 \%$ and $20 \%$ of the patients, respectively. An international, randomized phase II study evaluating the efficacy of cisplatin with or without cetuximab in the setting of triple-negative breast cancer is currently ongoing. Although promising, these initial data illustrate that the majority of patients with triple-negative breast cancer will benefit briefly, if at all, from EGFR inhibition; thus, rational strategies including EGFR inhibition are likely to require combining targeted agents.

\section{Src Inhibition: Dasatinib}

Gene expression profiling has suggested that basal-like breast cancers might be preferentially sensitive to inhibition of proto-oncogene, $S R C$. Dasatinib, a potent orally available inhibitor of Src-family kinases and other kinases with antiproliferative, antiosteoclastic, and antimetastatic activity, was recently studied in the setting of triplenegative $\mathrm{MBC}^{49}$ A phase II study reports a clinical benefit rate of $9.2 \%$ (2 PRs and 2 SDs) among 43 response-evaluable patients. Dose reduction from $100 \mathrm{mg}$ orally twice daily to 70 mg orally twice daily improved the toxicity profile. Although modest, encouraging singleagent activity was observed with dasatinib in patients with advanced triple-negative breast cancer. Because dramatic response to single-agent biologic agents is not expected, novel combinations of dasatinib and chemotherapy are warranted and are currently being explored.

\section{Histone Deacetylase Inhibitors}

Epigenetic mechanisms might play a role in the loss of ER- $\alpha$ in ER-negative breast tumors. Preclinical studies have shown that pharmacologic inhibition of these mechanisms (ie, DNA methyltransferase and HDAC inhibitors) result in re-expression of functional ER mRNA and protein. Specifically, treatment of 3 ER negative breast cancer cell lines (MDA-MB-231, MDA-MB-435, and Hs578t) and a xenograft model with an HDAC inhibitor (Scriptaid) resulted in both significant growth inhibition and reexpression of estrogen-responsive genes, namely PgR; a second study illustrated that this approach renders cells sensitive to hormonal manipulation with an aromatase inhibitor.44,58 A clinical study evaluating tamoxifen plus the HDAC inhibitor vorinostat (suberoylanilide hydroxamic acid; SAHA) in patients with heavily pretreated, endocrine-resistant ER-positive breast cancer illustrated 4 major responses and 5 patients with SD for $>12$ months. 50 The addition of an HDAC inhibitor to endocrine therapy in the setting of endocrine-resistant disease appears to restore sensitivity in select patients - a hypothesis being carried forward in the setting of ER-negative breast cancer. Currently, vorinostat is being evaluated in combination with capecitabine in the setting of advanced breast cancer. A second placebo-controlled study is evaluating the combination with carboplatin and albumin-bound paclitaxel with or without vorinostat in the neoadjuvant setting.

\section{MEK Inhibition in Preclinical Models}

Preclinical studies have illustrated that the small heat-shock protein, $\alpha \mathrm{B}$-crystallin, is commonly expressed (45\%) among basal-like breast tumors as determined by microarray analysis and independently predicts shorter survival. ${ }^{42}$ Interestingly, overexpression of $\alpha \mathrm{B}$ crystallin induces neoplastic changes in mammary acini (single layers of polarized, growtharrested mammary epithelial cells surrounded by a hollow lumen), transforms immortalized 
human mammary epithelial cells, and increases cell migration and invasion in vitro. Most strikingly, inhibitors of the MEK/ERK pathway, which is constitutively activated by $\alpha \mathrm{B}$ crystallin overexpression, suppresses the transformed mammary acinar phenotype, suggesting that MEK inhibitors might be an effective therapy for basal-like breast tumors expressing $\alpha \mathrm{B}$-crystallin. Although not yet available in the setting of advanced breast cancer, several MEK inhibitors are currently being tested in phase I trials.

\section{Conclusion}

In summary, triple-negative breast cancer represents a distinct subset of breast cancers exhibiting both a unique molecular profile and set of risk factors, an aggressive and early pattern of metastases, a relative lack of therapeutic targets, and a poor prognosis compared with other breast cancer subtypes. Commonly, but not uniformly, the clinical classification of triple-negative breast cancer is synonymous with the basal-like subtype as determined via cDNA micro-array analysis. As we gain a deeper understanding of the biologic processes driving triple-negative breast cancer, the arena of targeted therapeutic agents will continue to evolve, including strategies targeting the DNA repair enzyme PARP1 as well as EGFR, HDAC, angiogenesis, Src, and beyond. Continued research aimed at more fully characterizing the molecular and epidemiologic factors, as well as patterns of metastases observed among triple-negative breast cancers, will advance the development of prevention and treatment strategies aimed at improving outcomes for patients diagnosed with this aggressive disease.

\section{References}

1. Perou CM, Sørlie T, Eisen MB, et al. Molecular portraits of human breast tumours. Nature 2000;406:747-752. [PubMed: 10963602]

2. Sørlie T, Perou CM, Tibshirani R, et al. Gene expression patterns of breast carcinomas distinguish tumor subclasses with clinical implications. Proc Natl Acad Sci U S A 2001;98:10869-10874. [PubMed: 11553815]

3. Sørlie T, Tibshirani R, Parker J, et al. Repeated observation of breast tumor subtypes in independent gene expression data sets. Proc Natl Acad Sci U S A 2003;100:8418-8423. [PubMed: 12829800]

4. Swain, SM. Triple-negative breast cancer: metastatic risk and role of platinum agents. Paper presented at: 44th Annual Meeting of the American Society of Clinical Oncology; May 30-June 3, 2008; Chicago, IL.

5. Nielsen TO, Hsu FD, Jensen K, et al. Immunohistochemical and clinical characterization of the basal-like subtype of invasive breast carcinoma. Clin Cancer Res 2004;10:5367-5374. [PubMed: 15328174]

6. Livasy CA, Karaca G, Nanda R, et al. Phenotypic evaluation of the basal-like subtype of invasive breast carcinoma. Mod Pathol 2006;19:264-271. [PubMed: 16341146]

7. Bertucci F, Finetti P, Cervera N, et al. How basal are triple-negative breast cancers? Int J Cancer 2008;123:236-240. [PubMed: 18398844]

8. Cleator S, Heller W, Coombes RC. Triple-negative breast cancer: therapeutic options. Lancet Oncol 2007;8:235-244. [PubMed: 17329194]

9. Kreike B, van Kouwenhove M, Horlings H, et al. Gene expression profiling and histopathological characterization of triple-negative/basal-like breast carcinomas. Breast Cancer 2007;9:R65.

10. Carey LA, Perou CM, Livasy CA, et al. Race, breast cancer subtypes, and survival in the Carolina Breast Cancer Study. JAMA 2006;295:2492-2502. [PubMed: 16757721]

11. Matos I, Dufloth R, Alvarenga M, et al. p63, cytokeratin 5, and P-cadherin: three molecular markers to distinguish basal phenotype in breast carcinomas. Virchows Arch 2005;447:688-694. [PubMed: 16012853]

12. Narod SA. Modifiers of risk of hereditary breast and ovarian cancer. Nat Rev Cancer 2002;2:113123. [PubMed: 12635174] 
13. Narod SA, Foulkes WD. BRCA1 and BRCA2: 1994 and beyond. Nat Rev Cancer 2004;4:665676. [PubMed: 15343273]

14. Arnes JB, Brunet J-S, Stefansson I, et al. Placental cadherin and the basal epithelial phenotype of BRCA1-related breast cancer. Clin Cancer Res 2005;11:4003-4011. [PubMed: 15930334]

15. Foulkes WD, Stefansson IM, Chappuis PO, et al. Germline BRCA1 mutations and a basal epithelial phenotype in breast cancer. J Natl Cancer Inst 2003;95:1482-1485. [PubMed: 14519755]

16. Laakso M, Loman N, Borg Å, et al. Cytokeratin 5/14-positive breast cancer: true basal phenotype confined to BRCA1 tumors. Mod Pathol 2005;18:1321-1328. [PubMed: 15990899]

17. Lakhani SR, Reis-Filho JS, Fulford L, et al. Prediction of BRCA1 status in patients with breast cancer using estrogen receptor and basal phenotype. Clin Cancer Res 2005;11:5175-5180. [PubMed: 16033833]

18. Bauer KR, Brown M, Cress RD, et al. Descriptive analysis of estrogen receptor (ER)-negative, progesterone receptor (PR)-negative, and HER2-negative invasive breast cancer, the so-called triple-negative phenotype: a population-based study from the California Cancer Registry. Cancer 2007;109:1721-1728. [PubMed: 17387718]

19. Morris GJ, Naidu S, Topham AK, et al. Differences in breast carcinoma characteristics in newly diagnosed African-American and Caucasian patients: a single-institution compilation compared with the National Cancer Institute's Surveillance, Epidemiology, and End Results database. Cancer 2007;110:876-884. [PubMed: 17620276]

20. Millikan RC, Newman B, Tse C-K, et al. Epidemiology of basal-like breast cancer [published erratum in: Breast Cancer Res Treat 2008; 109:141]. Breast Cancer Res Treat 2008;109:123-139. [PubMed: 17578664]

21. Yang XR, Sherman ME, Rimm DL, et al. Differences in risk factors for breast cancer molecular subtypes in a population-based study. Cancer Epidemiol Biomarkers Prev 2007;16:439-443. [PubMed: 17372238]

22. Dent R, Trudeau M, Pritchard KI, et al. Triple-negative breast cancer: clinical features and patterns of recurrence. Clin Cancer Res 2007;13:4429-4434. [PubMed: 17671126]

23. Collett K, Stefansonn IM, Eide J, et al. A basal epithelial phenotype is more frequent in interval breast cancers compared with screen detected tumors. Cancer Epidemiol Biomarkers Prev 2005;14:1108-1112. [PubMed: 15894660]

24. Liedtke C, Mazouni C, Hess KR, et al. Response to neoadjuvant therapy and long-term survival in patients with triple-negative breast cancer. J Clin Oncol 2008;26:1275-1281. [PubMed: 18250347]

25. Smid M, Wang Y, Zhang Y, et al. Subtypes of breast cancer show preferential site of relapse. Cancer Res 2008;68:3108-3114. [PubMed: 18451135]

26. Heitz F, Harter P, Traut A, et al. Cerebral metastases (CM) in breast cancer (BC) with focus on triple-negative tumors. J Clin Oncol 2008;26(15 suppl):43s. (abstract 1010).

27. Lin NU, Claus E, Sohl J, et al. Sites of distant recurrence and clinical outcomes in patients with metastatic triple-negative breast cancer: high incidence of central nervous system metastases. Cancer 2008;113:2638-2645. [PubMed: 18833576]

28. Niwinska A, Murawska M. Brain metastases in breast cancer patients: differences in survival depending on biological subtype and RPA RTOG prognostic class. J Clin Oncol 2008;26(15 suppl):55s. (abstract 1056).

29. Bendell JC, Domchek SM, Burstein HJ, et al. Central nervous system metastases in women who receive trastuzumab-based therapy for metastatic breast carcinoma. Cancer 2003;97:2972-2977. [PubMed: 12784331]

30. Carey LA, Dees EC, Sawyer L, et al. The triple negative paradox: primary tumor chemosensitivity of breast cancer subtypes. Clin Cancer Res 2007;13:2329-2334. [PubMed: 17438091]

31. Rouzier R, Perou CM, Symmans WF, et al. Breast cancer molecular subtypes respond differently to preoperative chemotherapy. Clin Cancer Res 2005;11:5678-5685. [PubMed: 16115903]

32. Hayes DF, Thor AD, Dressler LG, et al. HER2 and response to paclitaxel in node-positive breast cancer. N Engl J Med 2007;357:1496-1506. [PubMed: 17928597] 
33. Hudis C, Citron M, Berry D, et al. Five year follow-up of INT C9741: dose-dense (DD) chemotherapy (CRx) is safe and effective. Breast Cancer Res Treat 2005;94 suppl 1:S20. (abstract 41).

34. Taniguchi T, Tischkowitz M, Ameziane N, et al. Disruption of the Fanconi anemia-BRCA pathway in cisplatin-sensitive ovarian tumors. Nat Med 2003;9:568-574. [PubMed: 12692539]

35. Turner N, Tutt A, Ashworth A. Hallmarks of 'BRCAness' in sporadic cancers. Nat Rev Cancer 2004;4:814-819. [PubMed: 15510162]

36. Carey LA, Rugo HS, Marcom PK, et al. TBCRC 001: EGFR inhibition with cetuximab added to carboplatin in metastatic triple-negative (basal-like) breast cancer. J Clin Oncol 2008;26(15 suppl): 43s. (abstract 1009).

37. Garber JE, Richardson A, Harris LN, et al. Neo-adjuvant cisplatin (CDDP) in "triple-negative" breast cancer (BC). Breast Cancer Res Treat 2006;100 suppl 1:S149. (abstract 3074).

38. O'Shaughnessy J, Weckstein DJ, Vukelja SJ, et al. Preliminary results of a randomized phase II study of weekly irinotecan/carboplatin with or without cetuximab in patients with metastatic breast cancer. Breast Cancer Res Treat 2007;106 suppl 1:S32. (abstract 308).

39. O'Shaughnessy J, Yoffe M, Osborne C, et al. Triple negative breast cancer: a phase 2, multicenter, open-label, randomized trial of gemcitabine/carboplatin (G/C), with or without BSI-201, a PARP inhibitor. Cancer Res 2009;69 suppl:194s. (abstract 2120).

40. Korsching E, Packeisen J, Agelopoulos K, et al. Cytogenetic alterations and cytokeratin expression patterns in breast cancer: integrating a new model of breast differentiation into cytogenetic pathways of breast carcinogenesis. Lab Invest 2002;82:1525-1533. [PubMed: 12429812]

41. Troester MA, Herschkowitz JI, Oh DS, et al. Gene expression patterns associated with p53 status in breast cancer. BMC Cancer 2006;6:276. [PubMed: 17150101]

42. Moyano JV, Evans JR, Chen F, et al. $\alpha \mathrm{B}$-crystallin is a novel oncoprotein that predicts poor clinical outcome in breast cancer. J Clin Invest 2006;116:261-270. [PubMed: 16395408]

43. Sparano JA, Gray R, Goldstein LJ, et al. GRB7-dependent pathways are potential therapeutic targets in triple-negative breast cancer. Cancer Res 2009;69 suppl:70s. (abstract 25).

44. Keen JC, Yan L, Mack KM, et al. A novel histone deacetylase inhibitor, scriptaid, enhances expression of functional estrogen receptor $\alpha(E R)$ in ER negative human breast cancer cells in combination with 5-aza 2'-deoxycytidine. Breast Cancer Res Treat 2003;81:177-186. [PubMed: 14620913]

45. Miller K, Wang M, Gralow J, et al. Paclitaxel plus bevacizumab versus paclitaxel alone for metastatic breast cancer. N Engl J Med 2007;357:2666-2676. [PubMed: 18160686]

46. Miles D, Chan A, Romieu G, et al. Randomised, double-blind, placebo-controlled, phase III study of bevacizumab (BV) with docetaxel (D) or docetaxel with placebo (PL) as first-line therapy for patients with locally recurrent or metastatic breast cancer (mBC): AVADO. J Clin Oncol 2008;26(15 suppl):1008s. (abstract LBA1011).

47. Kopetz S, Mita MM, Mok I, et al. First in human phase I study of BSI-201, a small molecule inhibitor of poly ADP-ribose polymerase (PARP) in subjects with advanced solid tumors. J Clin Oncol 2008;26(15 suppl):172s. (abstract 3577).

48. Mahany JJ Jr, Lewis N, Heath EI, et al. A phase IB study evaluating BSI-201 in combination with chemotherapy in subjects with advanced solid tumors. J Clin Oncol 2008;26(15 suppl):172s. (abstract 3579).

49. Finn RS, Bengala C, Ibrahim N, et al. Phase II trial of dasatinib in triple-negative breast cancer: results of study CA180059. Cancer Res 2009;69 suppl:242s. (abstract 3118).

50. Lacevic M, Minton SE, Schmitt ML, et al. Phase II trial of the HDAC inhibitor, vorinostat, in combination with tamoxifen for patients with advanced breast cancer who have failed prior antihormonal therapy. Breast Cancer Res Treat 2007;106 suppl 1:S117. (abstract 2097).

51. Citron ML, Berry DA, Cirrincione C, et al. Randomized trial of dose-dense versus conventionally scheduled and sequential versus concurrent combination chemotherapy as postoperative adjuvant treatment of node-positive primary breast cancer: first report of Intergroup trial C9741/Cancer and Leukemia Group B trial 9741 [published erratum in: J Clin Oncol 2003; 21:2226]. J Clin Oncol 2003;21:1431-1439. [PubMed: 12668651] 
52. Tentori L, Graziani G. Chemopotentiation by PARP inhibitors in cancer therapy. Pharmacol Res 2005;52:25-33. [PubMed: 15911331]

53. Bryant HE, Schultz N, Thomas HD, et al. Specific killing of BRCA2-deficient tumours with inhibitors of poly(ADP-ribose) polymerase [published erratum in: Nature 2007; 447:346]. Nature 2005;434:913-917. [PubMed: 15829966]

54. Farmer H, McCabe N, Lord CJ, et al. Targeting the DNA repair defect in BRCA mutant cells as a therapeutic strategy. Nature 2005;434:917-921. [PubMed: 15829967]

55. Foulkes WD, Brunet J-S, Stefansson IM, et al. The prognostic implication of the basal-like (cyclin Ehigh/p27low/p53+/glomeruloid-microvascular-proliferation+) phenotype of BRCA1-related breast cancer. Cancer Res 2004;64:830-835. [PubMed: 14871808]

56. O’Shaughnessy J, Osborne C, Pippen J, et al. Efficacy of BSI-201 a poly (ADP-ribose) polymerase-1 (PARP) inhibitor, in combination with gemcitabine/carboplatin $(\mathrm{G} / \mathrm{C})$ in patients with metastatic triple-negative breast cancer (TNBC): results of a randomized phase II trial. J Clin Oncol 2009;27 suppl:793s. (abstract 3).

57. Carey LA, Mayer E, Marcom PK, et al. TBCRC 001: EGFR inhibition with cetuximab in metastatic triple negative (basal-like) breast cancer. Breast Cancer Res Treat 2007;106 suppl 1:S32. (abstract 307).

58. Sabnis GJ, Gediya LK, Njar VCO, et al. HDAC inhibitors sensitize ER negative breast cancer cells to AIs. Breast Cancer Res Treat 2007;106 suppl 1:S117. (abstract 2096). 

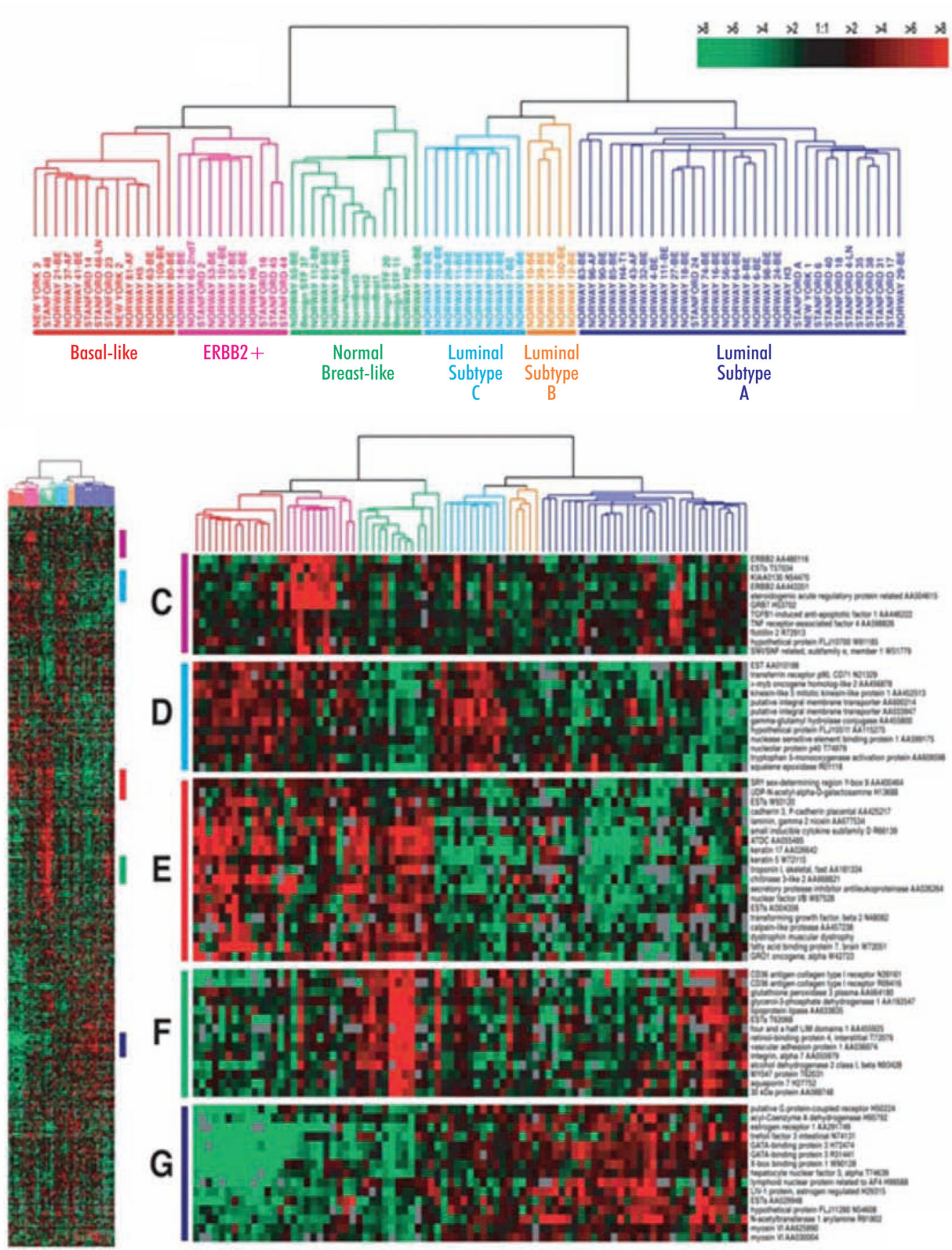

Figure 1.

Gene Expression Patterns of 85 Experimental Breast Tissues (78 Carcinomas, 3 Benign Tumors, and 4 Normal Tissues) Analyzed via Hierarchical Clustering Using the 476-cDNA Intrinsic Gene Set

Reproduced with permission. Sørlie T, Perou CM, Tibshirani R, et al. Gene expression patterns of breast carcinomas distinguish tumor subclasses with clinical implications. Proc Natl Acad Sci U S A 2001; 98:10869-74. Copyright 2001 National Academy of Sciences, U.S.A. 
A Survival

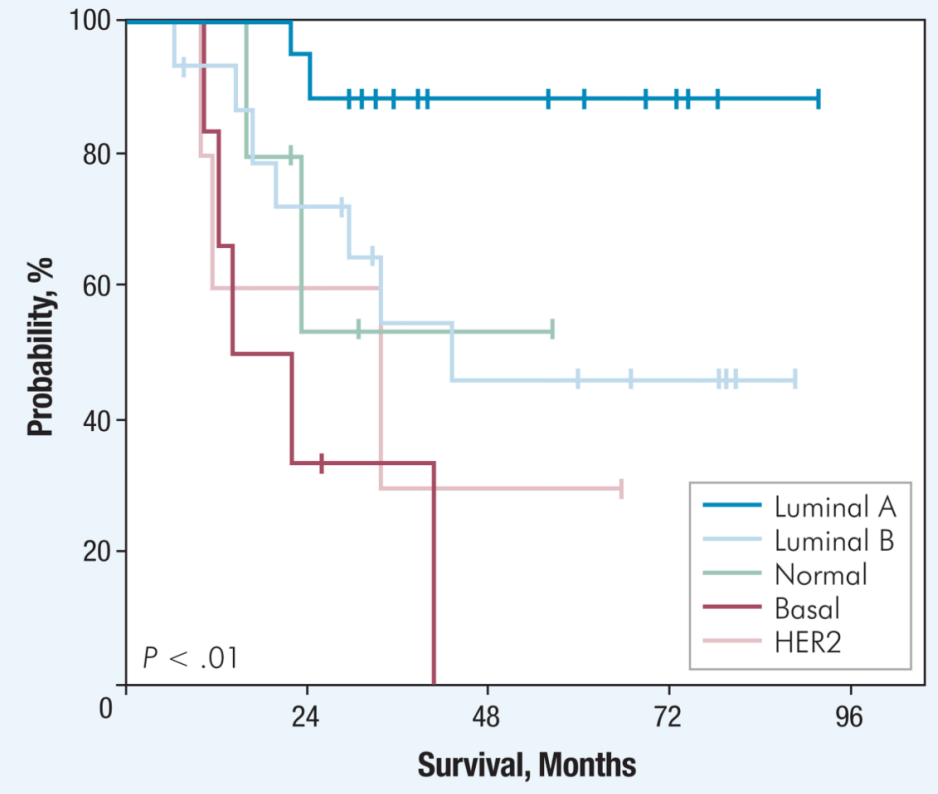

B Recurrence-Free Survival

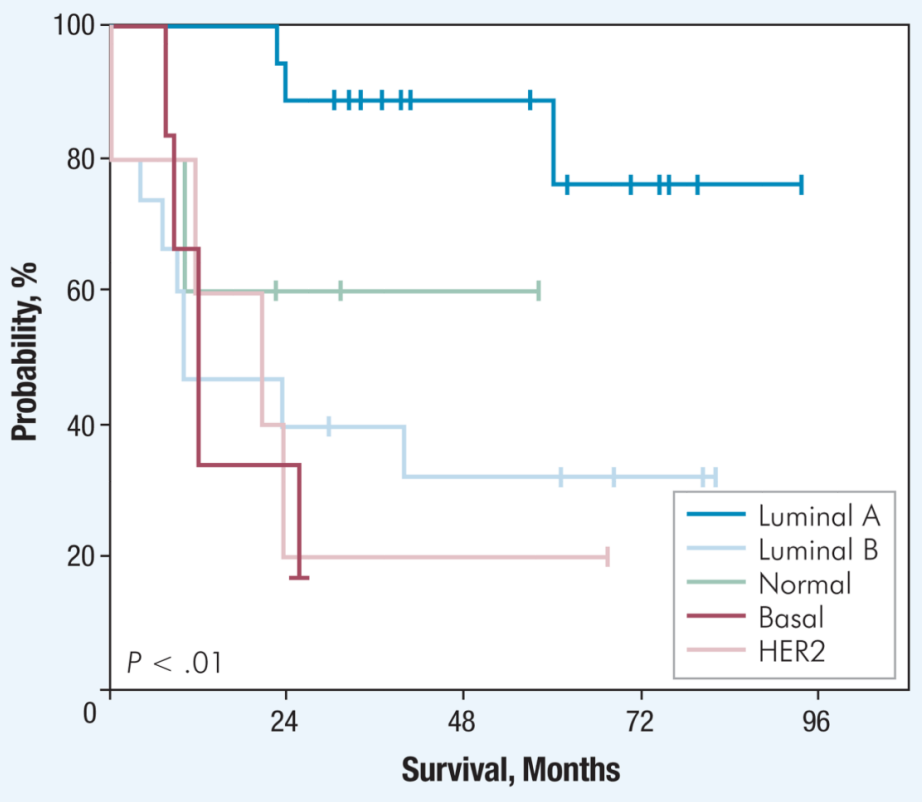

Figure 2.

Prognosis as Defined via Intrinsic Subtypes

Reproduced with permission. Sø rlie T, Perou CM, Tibshirani R, et al. Gene expression patterns of breast carcinomas distinguish tumor subclasses with clinical implications. Proc Natl Acad Sci U S A 2001; 98:10869-74. Copyright 2001 National Academy of Sciences, U.S.A. 
Table 1

Molecular and Pathologic Features of Triple-Negative Basal-like Breast Cancer

\begin{tabular}{c|c}
\hline $\begin{array}{c}\text { Immunophenotypic } \\
\text { Characteristics }\end{array}$ & Pathologic Characteristics \\
\hline Estrogen receptor negative & Grade III \\
\hline HER2 negative & High proliferation rate \\
\hline P-cadherin negative & Nuclear pleomorphism \\
\hline p63 negative & Pushing borders of invasion \\
\hline HER1 (EGFR) positive & Geographic necrosis \\
\hline Cytokeratin 5/6 positive & p53 mutations $(\approx 80 \%)^{2}$ \\
\hline c-Kit positive & BRCA1 germline mutations \\
\hline Vimentin positive & Ductal and metaplastic histology \\
\hline
\end{tabular}

Majority across studies. 5,6 
Table 2

Risk Factors Associated with Basal-like (Estrogen Receptor/HER2-Negative/HER1 and/or Cytokeratin 5/6Positive) Breast Cancer in the Population-Based Carolina Breast Cancer Study ${ }^{20}$

\begin{tabular}{c}
\hline $\begin{array}{c}\text { Risk Factors Associated with } \\
\text { Basal-like/Triple-Negative Breast Cancer }\end{array}$ \\
\hline African American race \\
\hline Premenopausal status \\
\hline Increasing parity \\
\hline Younger age at first-term pregnancy \\
\hline Shorter duration of breast feeding \\
\hline Use of lactation-suppression techniques \\
\hline Elevated waist-to-hip ratio (both pre- and postmenopausal women)
\end{tabular}


Table 3

Therapeutic Strategies, Confirmed and in Development, for Triple-Negative Breast Cancer

\begin{tabular}{c|c}
\hline $\begin{array}{c}\text { Therapeutic } \\
\text { Strategy or Target }\end{array}$ & Status of Development \\
\hline $\begin{array}{c}\text { Anthracycline-/taxane-based } \\
\text { chemotherapy }\end{array}$ & $\begin{array}{c}\text { Proven efficacy, phase II/III } \\
\text { clinical trials } 30-32,51\end{array}$ \\
\hline Platinum agents & $\begin{array}{c}\text { Active agents, phase II } \\
\text { clinical trials } 36-38\end{array}$ \\
\hline EGFR inhibition & $\begin{array}{c}\text { Modest activity, phase II } \\
\text { clinical trials } 36,38\end{array}$ \\
\hline Antiangiogenesis & $\begin{array}{c}\text { Efficacy in subset analysis, } \\
\text { phase III trials } 45,46\end{array}$ \\
\hline PARP1 inhibition & $\begin{array}{c}\text { Safety illustrated, efficacy results } \\
\text { anticipated, phase I/II trials } 39,47,48\end{array}$ \\
\hline Src inhibition & Modest activity, phase II trials 49 \\
\hline HDAC inhibition & $\begin{array}{c}\text { Activity in preclinical studies, } \\
\text { early clinical development } 44,50\end{array}$ \\
\hline MEK inhibition & Activity in preclinical studies 42 \\
\hline
\end{tabular}

Abbreviations: EGFR = epidermal growth factor receptor; HDAC = histone deacetylase; PARP1 = poly (adenosine diphosphate-ribose $)$ polymerase-1 